\title{
The influence of variations in eating disorder-related symptoms on processing of emotional faces in a non- clinical female sample: an eye-tracking study
}

Emma Sharpe ${ }^{a}$, Deborah J. Wallis ${ }^{a^{*}}$, Nathan Ridout ${ }^{\mathrm{b}}$

${ }^{a}$ Loughborough University Centre for Eating Disorders, School of Sport, Exercise and Health Sciences, Loughborough University, Loughborough, LE11 3TU, UK.

Email: E.Sharpe@lboro.ac.uk; Debbie.Wallis@dmu.ac.uk

${ }^{b}$ Department of Psychology, School of Life and Health Sciences, Aston University, Birmingham, B4 7ET, UK.

Email: n.ridout@aston.ac.uk

* Corresponding author (present address):

Dr Deborah J. Wallis

School of Applied Social Sciences

Hawthorn Building

De Montfort University

Leicester

LE1 9BH

Tel: 01162577471

Email: debbie.wallis@dmu.ac.uk 


\begin{abstract}
This study aimed to: i) determine if the attention bias towards angry faces reported in eating disorders generalises to a non-clinical sample varying in eating disorderrelated symptoms; ii) examine if the bias occurs during initial orientation or later strategic processing; and iii) confirm previous findings of impaired facial emotion recognition in non-clinical disordered eating. Fifty-two females viewed a series of face-pairs (happy or angry paired with neutral) whilst their attentional deployment was continuously monitored using an eye-tracker. They subsequently identified the emotion portrayed in a separate series of faces. The highest $(n=18)$ and lowest scorers $(n=17)$ on the Eating Disorders Inventory (EDI) were compared on the attention and facial emotion recognition tasks. Those with relatively high scores exhibited impaired facial emotion recognition, confirming previous findings in similar non-clinical samples. They also displayed biased attention away from emotional faces during later strategic processing, which is consistent with previously observed impairments in clinical samples. These differences were related to drive-for-thinness. Although we found no evidence of a bias towards angry faces, it is plausible that the observed impairments in emotion recognition and avoidance of emotional faces could disrupt social functioning and act as a risk factor for the development of eating disorders.
\end{abstract}

\title{
Key Words
}

Attention, eating disorders, eye-movements, disordered eating, drive-for-thinness. 


\section{Introduction}

Eating disorders such as anorexia nervosa (AN) and bulimia nervosa (BN) are associated with deficits in social and emotional functioning. For example, there is a body of evidence demonstrating that patients with eating disorders exhibit impaired facial emotion recognition in comparison to healthy controls (Jänsch et al., 2009; Kucharska-Pietura et al., 2004; Pollatos et al., 2008). This deficit is also evident when participants are restricted to viewing just the eyes (Harrison et al., 2010a; 2010b; Russell et al., 2009). Furthermore, similar impairments have been observed in non-clinical participants who could be considered 'at risk' of developing eating disorders (Jones et al., 2009; Ridout et al., 2010; 2012). However, it should be noted that other studies have found no evidence of impaired facial emotion recognition in patients with eating disorders. For example, Cardi et al. (2015) reported that, in comparison to healthy controls, patients with AN and BN were not impaired in their ability to recognise emotion from video clips. Furthermore, Brewer et al. (2015) reported that emotion recognition deficits in a group of patients with eating disorders (AN and BN) were related to concomitant alexithymia and not eating disorder symptomology. Nevertheless, a recent meta-analysis conducted by Caglar-Nazali et al. (2014) did show evidence of impaired emotion recognition in patients with eating disorders, although this was a small effect size and became non-significant once the moderator variables of group and task type were accounted for in meta-regression. Taken together there is evidence of impaired facial emotion recognition in participants with disordered eating although the stability of this deficit may depend on the type of sample examined and emotion recognition task employed.

In addition to deficits in facial emotion recognition, there is evidence that patients with eating disorders display attentional biases for socially relevant stimuli. For example, McManus et al. (1996) found evidence of biased attention towards emotional words, particularly those connoting threat. Harrison et al. (2010a) reported that this bias generalised to faces. They found that, compared with healthy controls, patients with eating disorders experienced greater interference from angry faces on a face-variant of the Stroop task. This finding has subsequently been replicated (Kanakam et al., 2013) and has been found to persist in recovered patients (Harrison et al., 2010b). Similarly, Cardi et al. (2012) utilised a dot-probe task and reported that patients with eating disorders exhibited an initial orientation bias towards rejecting 
faces, subsequent problems in disengaging their attention from these faces, and a tendency to avoid attending to accepting faces. Harrison et al. (2010b) has suggested that biased attention for angry faces might be a possible trait vulnerability factor for eating disorders. However, despite this evidence, there is also a growing body of work showing that individuals with eating disorders have a tendency to avoid attending to emotional faces (e.g. Davies et al., 2014; Rhind et al., 2014). For example, Cardi et al. (2015) reported that patients with AN and BN looked away from video clips of emotional expressions of happiness, sadness and anger significantly more often than did healthy controls. They concluded that this tendency could contribute to the deficits in social and emotional functioning that have been observed in patients with eating disorders.

It has yet to be established if biased attention towards angry faces generalises to those with non-clinical levels of disordered eating. Traditionally, research designed to identify cognitive impairments in eating disorders has focused on investigating clinical populations, which has made it particularly difficult to separate the causal and maintaining factors implicated in disordered eating (Stice, 2002). Therefore, a focus on non-clinical levels of eating disorder-related symptoms within the general population may help to distinguish between the factors implicated in the development of eating-related psychopathology and those which maintain the disorder. Overall, work examining individuals without a clinical diagnosis might prove valuable in elucidating the nature of symptom development and progression. It is known that those in the early stages of eating disorder development are the most likely to respond to treatment (Gordon, 2000). Therefore, identifying the factors involved in the development of disordered eating at the non-clinical stage may help to inform early intervention strategies.

To date, the majority of work assessing attentional bias in participants with disordered eating has tended to use variants of either the Stroop or dot probe tasks. However, as noted by Giel et al. (2011), these methods lack the appropriate temporal resolution to clearly delineate the nature of attentional biases. Greater response latencies on these tasks might reflect a bias towards a stimulus (orientation) or problems moving attention away from it (disengagement). Orientation is thought to be an automatic process that occurs early in the temporal processing of 
a stimulus, whereas disengagement is thought to be a later, strategically controlled, process. Studies using the dot-probe task utilise different presentation times to try and distinguish between these two processes. However, this task only provides a 'snap shot' of the location of attention at a given moment in time, which is a notable limitation as the time chosen for this sampling can influence the direction of bias observed (Cooper and Langton, 2006). Eye-tracking technology enables the continuous measurement of eye-movements using a high temporal resolution, thus it is able to overcome the limitations of previous studies. Eye-tracking has been used successfully to investigate the processing of salient cues in different participant groups (Caivo et al., 2007; Mogg et al., 2003; Nummenmaa et al., 2006). Notably, it has been used successfully to investigate attentional biases to food- and bodyrelated cues in participants with eating disorders (Giel et al, 2011) and nonclinical participants with relatively high scores on measures of disordered eating (Gao et al., 2011; Hewig et al., 2008). With this in mind, the current study utilised eye-tracking in order to establish if healthy participants who could be considered 'at risk' of developing an eating disorder would exhibit biased attention towards angry faces. $A$ further aim was to determine if the bias related to initial orientation or prolonged engagement (delayed disengagement). We also aimed to confirm previous findings (Jones et al., 2009; Ridout et al., 2010; 2012) of impaired facial emotion recognition in healthy participants with relatively high levels of eating disorder-related symptoms.

In the present study, healthy participants with relatively high or low scores on the eating disorder-related subscales of the Eating Disorders Inventory (EDI; Garner, 1991) were presented with pairs of faces (angry-neutral or happy-neutral) and asked to inspect them under free viewing conditions (as in Giel et al., 2011). Eyemovements were continuously monitored using an eye-tracker. Following this task participants were presented with a separate series of faces and asked to identify the emotion portrayed (eye movements were not collected during this task). Given previous findings of an attention bias to angry faces in patients with eating disorders (Harrison et al., 2010a; b), it was hypothesised that individuals with relatively high EDI scores would demonstrate a greater tendency to initially fixate on the angry faces than would low EDI scorers. It is also expected that those with higher EDI scores would spend longer during these initial fixations looking at the angry faces than would low scorers. Both of these findings would suggest biased orienting to 
these threatening stimuli. It was also expected that those with relatively high EDI scores would have difficulties disengaging from angry faces (i.e. longer total gaze duration times on the angry faces) than would low EDI scorers, which would suggest a problem in disengaging attention from threat. On the facial emotion recognition task, it was expected that those with relatively high EDI scores would correctly identify fewer emotional expressions than would the low EDI scorers, and this deficit would be particularly evident for negative emotions. In the analysis of the continuous data (with the total sample), it was predicted that the percentage and duration of initial fixations on angry expressions and the total gaze duration on angry faces would be positively related to scores on the EDI subscales of drive-for-thinness, body dissatisfaction and bulimia. Finally, it was expected that the percentage of expressions correctly recognised would be negatively related to scores on the EDI subscales, particularly drive for thinness.

\section{Method}

\subsection{Participants}

Fifty two female volunteers ${ }^{1}$ aged between 18 and 29 (mean age=22.13, SD=2.48) took part in the study. A tertile split was conducted on the participants' scores on the eating disorder-related subscales of the Eating Disorders Inventory (EDI; Garner, 1991). The highest scoring $(n=18)$ and lowest scoring participants $(n=17)$ were included in a group analysis of the face processing data ${ }^{2}$. The mean age of the high EDI group was 21.17 (SD=2.15) and of the low EDI group was 21.65 (SD=2.98). Further details of the two groups can be found in Table 1. All 52 participants were included in the continuous analysis of the data. This study was approved by the research ethics committee of Loughborough University.

\subsection{Measures}

Eating psychopathology was assessed using the three eating disorder-related

\footnotetext{
${ }^{1}$ All participants reported no history of an eating disorder. However, the EDI scores of two participants were within the range of EDI scores observed in patients with AN (Garner, 1991), which suggests they could potentially meet the diagnostic criteria for AN. This was considered during the analysis of face processing data (see footnote 5).

2 Power calculations using G*Power revealed that a total sample of 34 participants is sufficient to detect a large effect size in the critical two-way analyses. However, the study may be underpowered to detect a medium effect size. Furthermore, a sample of 50 participants was identified as appropriate to reliably detect medium effect sizes using multiple regression with up to five predictors.
} 
subscales of the Eating Disorder Inventory (EDI; Garner, 1991). This continuous measure consists of 23 items assessing drive for thinness ( 7 items), bulimia ( 7 items) and body dissatisfaction ( 9 items). All items are scored from 0 to 3 (the most extreme response scoring ' 3 ', the next two scoring ' 2 ' and ' 1 ' respectively, and the final three all scoring ' 0 '). The score on each subscale is the sum of the responses to all items on the subscale. The total EDI score (ranging between 0 and 69) is obtained by summing the scores from each of the three subscales, with higher scores equating to more severe eating-related psychopathology. Previously, Bulimia subscale scores above two have been regarded as high (e.g. Waller et al., 1996). For the DFT subscale, scores above seven are considered high, while those below two are seen as low (e.g. Ben-Tovim and Walker, 1991). Previous work by Ridout et al. (2010) revealed that scores above 15 on the body dissatisfaction can be considered high relative to the published norms for nonclinical female samples (Garner, 1991). This measure has been used successfully in student populations (Laquatra and Clopton, 1994; Quinton and Wagner, 2005; Ridout et al., 2010; 2012). Reliability was confirmed in the current sample, as Cronbach's alpha values of $0.89,0.88$ and 0.92 were found for the Drive for thinness, Bulimia, and Body dissatisfaction subscales respectively.

The 21-item Beck Depression Inventory (BDI-II; Beck et al., 1996) was used to assess the presence and severity of depression within the participant sample. Scores on this self-report measure range from 0-63 with higher scores equating to more severe depression. This scale is widely used in the literature, and demonstrates high internal consistency with an alpha coefficient of 0.93 for nonpsychiatric populations (Beck et al., 1996). A high Cronbach's alpha score of 0.92 was also found in the present study, suggesting a high degree of reliability.

The 20-item Toronto Alexithymia Scale (TAS-20; Bagby et al., 1994) was used to assess the presence and severity of alexithymia within the current sample. This selfreport measure consists of the three subscales "Difficulty Identifying Feelings", "Difficulty Describing Feelings", and an "Externally Oriented (or concrete) Thinking Style". Scores range from 20 to 100 with higher scores reflecting greater levels of alexithymia. Previous research has shown the TAS-20 to be a valid measure of alexithymia, which demonstrates high internal consistency (Cronbach's alpha $=0.81$ ) when investigating student populations (Bagby et al., 1994). The reliability of this 
measure was confirmed in the current sample, with Cronbach's alpha values of 0.86 , 0.93 and 0.80 for the Difficulty Identifying Feelings, Difficulty Describing Feelings and Externally Oriented Thinking respectively.

The 20-item trait subscale of the State-Trait Anxiety Inventory (STAI-T; Spielberger et al., 1970) was used to assess dispositional tendencies towards anxiety within the current sample. Scores on this self-report measure range from 20 to 80, with higher scores indicating more severe levels of trait anxiety. This measure has been used extensively within non-clinical groups with a high degree of reliability; Cronbach's alpha coefficients have been reported in the range of 0.85-0.95 (Spielberger et al., 1970). The reliability of this measure was confirmed in the current sample, with a Cronbach's alpha score of 0.96 .

\subsection{Facial Stimuli}

\subsubsection{Facial Attention task}

A set of 36 colour photographs of twelve individuals ( 6 males, 6 females) portraying three different facial expressions (happiness, anger and neutral affect) was drawn from the BU-3DFE database ${ }^{3}$ (Yin et al., 2006) for use in the facial attention task. The three photographs from each individual were combined to produce two pairs (neutral-happy and neutral-angry), which resulted in a total of 24 stimulus pairs. The individual pictures from each pair were $12 \mathrm{~cm} \times 13 \mathrm{~cm}$ and were displayed in two opposing corners of the computer screen (top left/bottom right or top right/bottom left) against a white background with their innermost corner located $1.5 \mathrm{~cm}$ from the screen's centre. The location (left/right) of emotional and neutral stimuli was fully counterbalanced across trials. This procedure is consistent with Giel et al. (2011).

\subsubsection{Facial Emotion Recognition task}

An additional set of 14 faces ( 7 male, 7 female) portraying different emotional expressions (happiness, sadness, anger, fear, surprise, disgust and neutral affect) was drawn from the BU-3DFE for use in the facial emotion recognition task.

\footnotetext{
${ }^{3}$ These 3D stimuli were produced using computer manipulations of 2D photographs of posed facial expressions. The authors reported an overall accuracy rate of $96 \%$ for the recognition of the emotional expressions portrayed.
} 


\subsection{Apparatus}

Gaze data were collected using Tobii 1750 eye-tracker and analysed using ClearView analysis software. This technology uses a non-invasive infrared-based binocular eye-tracking technique that does not require the participant to be constrained using a chin rest or bite bar, as it compensates for head movements when calculating the direction and location of gaze. The data were collected at a sampling rate of $50 \mathrm{~Hz}$ with a spatial acuity of $0.25^{\circ}$ to $0.5^{\circ}$. Binocular averaging was used to ensure that drift was maintained below $0.5^{\circ}$. All stimuli were displayed on a 17 inch computer screen with a resolution of $1280 \times 1024$ pixels.

\subsection{Procedure}

After providing written informed consent participants completed a general demographic questionnaire (to report age and any past/current health problems), followed by the EDI, BDI-II, TAS-20 and STAI-T. During the facial attention task participants were seated on a sturdy chair with a fixed back and viewed the screen from a distance of approximately $60 \mathrm{~cm}$. In order to ensure optimum gaze data quality the eye-tracker was calibrated for each individual (using a standardised 9point calibration procedure) prior to the beginning of the facial attention task.

Each trial of the facial attention task consisted of a fixation cross (presented centrally for $2000 \mathrm{msec}$ ), followed by a pair of faces which was displayed for $3000 \mathrm{msec}$. Participants were asked to look at the faces on screen as if they were watching a television programme (free viewing), but were instructed to return their focus to the central fixation cross whenever it appeared. The 24 stimulus pairs were presented in a new randomised order for each participant. During the facial emotion recognition task, participants were presented with a random sequence of the fourteen faces and asked to identify the emotion displayed (forced choice from a set of emotion labels). They also rated the pleasantness of each picture using a visual analogue scale from 0 (not at all pleasant) to 100 (extremely pleasant).

\subsection{Gaze Data}

Raw gaze data were condensed using ClearView analysis software. Following Giel et al. (2011) fixations with a duration (in milliseconds) of 3 standard deviations above the individual participant's mean were deemed outliers and were excluded $(4.7 \%$ of 
total fixation data). The emotional and neutral facial expressions presented on each trial were defined as areas of interest (AOI). In line with Giel et al. (2011), three key variables were extracted: the location of the initial fixation after trial onset (i.e. percentage of first fixations on each $\mathrm{AOI}$ ); the duration of this fixation (in milliseconds); and the total gaze duration (in milliseconds) on each $\mathrm{AOI}$ across the entire trial. The first two variables are thought to reflect initial orienting of attention, with the location indicating facilitated attention to a particularly salient stimulus and the duration of this initial fixation reflecting the depth of processing. The total duration of gaze on each $\mathrm{AOI}$ is thought to reflect the cognitive load associated with processing a stimulus, or interest in what is presented at the location. However, this metric can also be interpreted in terms of motivational processes such as aversion and reward, with increased gaze durations indicating attentional engagement and reduced gaze lengths signifying avoidance. A direction bias score was calculated for the happy and angry faces by subtracting the percentage of initial fixations on neutral faces from the percentage of initial fixations on the emotional faces. Positive scores reflect a greater tendency to initially attend to the emotional expressions. Similarly, an initial fixation duration bias score was also calculated by subtracting the duration of initial fixations on the neutral faces from the duration of initial fixations on the emotional faces. Positive scores indicate longer initial fixations on the emotional faces compared to the neutral. Finally, a gaze duration bias score was calculated by subtracting the total gaze duration on the neutral faces from the total gaze duration on the emotional faces. Positive scores suggest that participants spent longer looking at the emotional expressions, whereas negative scores indicate that they spent longer fixating on neutral faces, which could be interpreted as avoidance of the emotional faces.

\subsection{Data Analysis}

Independent t-tests were used to assess the significance of any differences between the high and low EDI scorers in age, eating psychopathology (EDI scores), depression (BDI-II scores), alexithymia (TAS-20 scores) and trait anxiety (STAI-T scores). Differences in initial orienting (direction bias score and initial fixation duration bias score) and subsequent engagement (gaze duration bias score) were 
analysed using separate 2 (EDI group) $\times 2$ (emotion) mixed factorial ANOVA, with EDI group (high vs low scorers) as the between subjects factor and emotion (angry vs happy expressions) as the within subjects factor. Percentage of facial expressions correctly recognised during the facial emotion recognition task were analysed using a 2(group) $\times 7$ (emotion) mixed factorial ANOVA, with EDI group (high vs low scorers) as the between subjects factor and emotion (happy vs sad vs angry vs disgusted vs surprised vs fearful vs neutral affect) as the within subjects factor. Correlational analyses with the whole sample were conducted to assess the influence of mood, alexithymia and eating psychopathology on face processing. Where appropriate, multiple regression was used to determine the relative importance of the eating disorder-related symptoms, mood, and alexithymia in accounting for variations in face processing. All tests were two-tailed with an alpha level of 0.05 unless otherwise stated.

\section{Results}

\subsection{Participant characteristics ${ }^{4}$}

Analysis of participant characteristics (see Table 1) revealed that the high and low EDI groups did not differ significantly in age or on the measures of alexithymia (TAS$20)$ and depression (BDI-II). However, the high EDI group scored significantly higher than those in the low EDI group on all three subscales of the EDI. They were also significantly more trait anxious than were the low EDI group.

\footnotetext{
${ }^{4}$ Participants' scores on the eating disorders inventory were skewed and there were problems concerning homogeneity of variance, which violate parametric assumptions. Therefore, we conducted non-parametric Mann Whitney tests to determine the significance of any group differences on this measure.
} 
Table 1: Participant characteristics (group means \pm standard deviations)

\begin{tabular}{lcccc}
\hline & $\begin{array}{c}\text { Low EDI } \\
(\mathbf{n}=\mathbf{1 7})\end{array}$ & $\begin{array}{c}\text { High EDI } \\
(\mathbf{n}=\mathbf{1 8})\end{array}$ & $\mathbf{t}$ & $\boldsymbol{P}$ \\
& $21.65(2.98)$ & $22.17(2.15)$ & 0.61 & 0.556 \\
\hline Age & $41.65(16.16)$ & $48.44(14.90)$ & 1.3 & 0.204 \\
Alexithymia (Total TAS-20) & $5.53(4.82)$ & $10.39(9.97)$ & 1.90 & 0.074 \\
Depression (BDI) & $35.29(13.83)$ & $45.28(9.76)$ & 2.48 & 0.018 \\
Trait Anxiety (STAI-T) & & & $\mathrm{U}$ & $p$ \\
\hline Eating Disorders Inventory (EDI) & & $18.00(8.51)$ & 306 & $<0.001$ \\
\hline EDI Total & $0.35(0.49)$ & $6.22(4.95)$ & 296.5 & $<0.001$ \\
Drive for Thinness & $0.06(0.28)$ & $1.56(2.64)$ & 214.5 & 0.041 \\
Bulimia & $0.06(0.24)$ & $10.22(3.8)$ & 306 & $<0.001$ \\
\hline Body Dissatisfaction & $0.24(0.44)$ & & & \\
\hline
\end{tabular}

\subsection{Attentional Orienting (Facial Attention Task)}

Analysis of the participants' direction bias scores (presented in Table 2) revealed no significant main effects of emotion or group, $p=0.99$ and $p=0.35$ respectively, and no significant group $x$ emotion interaction; $F(1,33)=1.71, p=0.20 ; \eta^{2} p=0.05$. Analysis of the participants' initial fixation duration bias scores (presented in Table 2) revealed no significant main effect of emotion or group $x$ emotion interaction; $p=0.63$ and $p=0.87$ respectively. Although the low EDI group spent longer looking at the emotional expressions (mean $=255.02 \mathrm{~ms} ; \mathrm{SD}=533.67$ ) than did high EDI group (mean=116.26 ms; $S D=278.33$ ) this difference was not significant; $F(1,33)=1.4$, $p=0.245 ; \eta^{2}=0.041$. 
Table 2: Mean direction bias score for initial fixation (\% difference) ${ }^{\mathrm{a}}$ and duration bias score (ms) ${ }^{b}$ for angry and happy emotional expressions in high and low EDI individuals (standard deviations are presented in parentheses)

\begin{tabular}{|c|c|c|c|c|c|c|}
\hline & \multicolumn{2}{|c|}{$\begin{array}{l}\text { Low EDI } \\
(n=17)\end{array}$} & \multicolumn{2}{|c|}{$\begin{array}{l}\text { High EDI } \\
(n=18)\end{array}$} & \multicolumn{2}{|c|}{$\begin{array}{c}\text { Total } \\
(n=35)\end{array}$} \\
\hline & Angry & Happy & Angry & Happy & Angry & Happy \\
\hline $\begin{array}{l}\text { Direction Bias Score } \\
\text { (DBS) for Initial Fixation }^{(\% \text { difference })^{a}}\end{array}$ & $\begin{array}{l}-3.95^{e} \\
(17.89)\end{array}$ & $\begin{array}{l}-7.44^{\mathrm{e}} \\
(19.15)\end{array}$ & $\begin{array}{l}-12.77^{e} \\
(14.13)\end{array}$ & $\begin{array}{l}-9.30^{e} \\
(21.78)\end{array}$ & $\begin{array}{l}-8.36^{d} \\
(16.01)\end{array}$ & $\begin{array}{l}-8.37^{d} \\
(20.47)\end{array}$ \\
\hline Total DBS & \multicolumn{2}{|c|}{$-5.70^{c}(18.52)$} & \multicolumn{2}{|c|}{$-11.04^{c}(17.96)$} & \multicolumn{2}{|c|}{$-8.37(18.24)$} \\
\hline $\begin{array}{l}\text { Initial Fixation Duration } \\
\text { (IFD) Bias Score } \\
\text { (milliseconds) }^{\mathrm{b}}\end{array}$ & $\begin{array}{c}270.2^{\mathrm{h}} \\
(337.20)\end{array}$ & $\begin{array}{c}239.7^{\mathrm{h}} \\
(730.13)\end{array}$ & $\begin{array}{c}146.7^{\mathrm{h}} \\
(239.24)\end{array}$ & $\begin{array}{c}85.7^{\mathrm{h}} \\
(317.42)\end{array}$ & $\begin{array}{l}208.45^{g} \\
(288.22)\end{array}$ & $\begin{array}{c}162.7^{g} \\
(523.78)\end{array}$ \\
\hline Total IFD bias score & \multicolumn{2}{|c|}{$254.95^{f}(533.67)$} & \multicolumn{2}{|c|}{$116.2^{f}(278.33)$} & \multicolumn{2}{|c|}{$185.58(406)$} \\
\hline
\end{tabular}

${ }^{a}$ A direction bias score was calculated by subtracting the percentage of initial fixations on emotional faces (both angry and happy) from the percentage of initial fixations on the neutral faces. Positive scores reflect a tendency to initially pay attention to emotional expressions.

${ }^{b}$ An initial fixation duration bias score was calculated by subtracting the duration of initial fixations on the emotional faces (both angry and happy) from the duration of initial fixations on the neutral faces. Positive scores reflect a tendency to initially fixate longer on emotional faces over neutral ones.

c-h all tests $p>0.05$ 


\subsection{Attentional Engagement (Facial Attention Task)}

Analysis of the gaze duration bias scores (presented in Figure 1) revealed no significant main effect of emotion or group $x$ emotion interaction; $p=0.067$ and $p=0.97$ respectively. However, the difference between the duration bias scores of the low EDI group (Mean=182.86 ms, SD=533.57) and high EDI group (Mean= $-403.73 \mathrm{ms,}$ $S D=908.06)$ was significant; $F(1,33)=5.81, p=0.022 ; \eta^{2}{ }_{p}=0.15$, which suggests that low scorers spent longer looking at the emotional faces than neutral, whereas the high EDI group spent longer looking at the neutral faces compared to emotional.

\section{Figure 1: Gaze duration bias scores for angry and happy faces in low and high EDI groups (error bars show \pm one standard error)}

\footnotetext{
a Separate gaze duration bias scores were calculated for angry and happy expression by subtracting the total gaze duration on the emotional face from the total gaze duration on the neutral face.
}

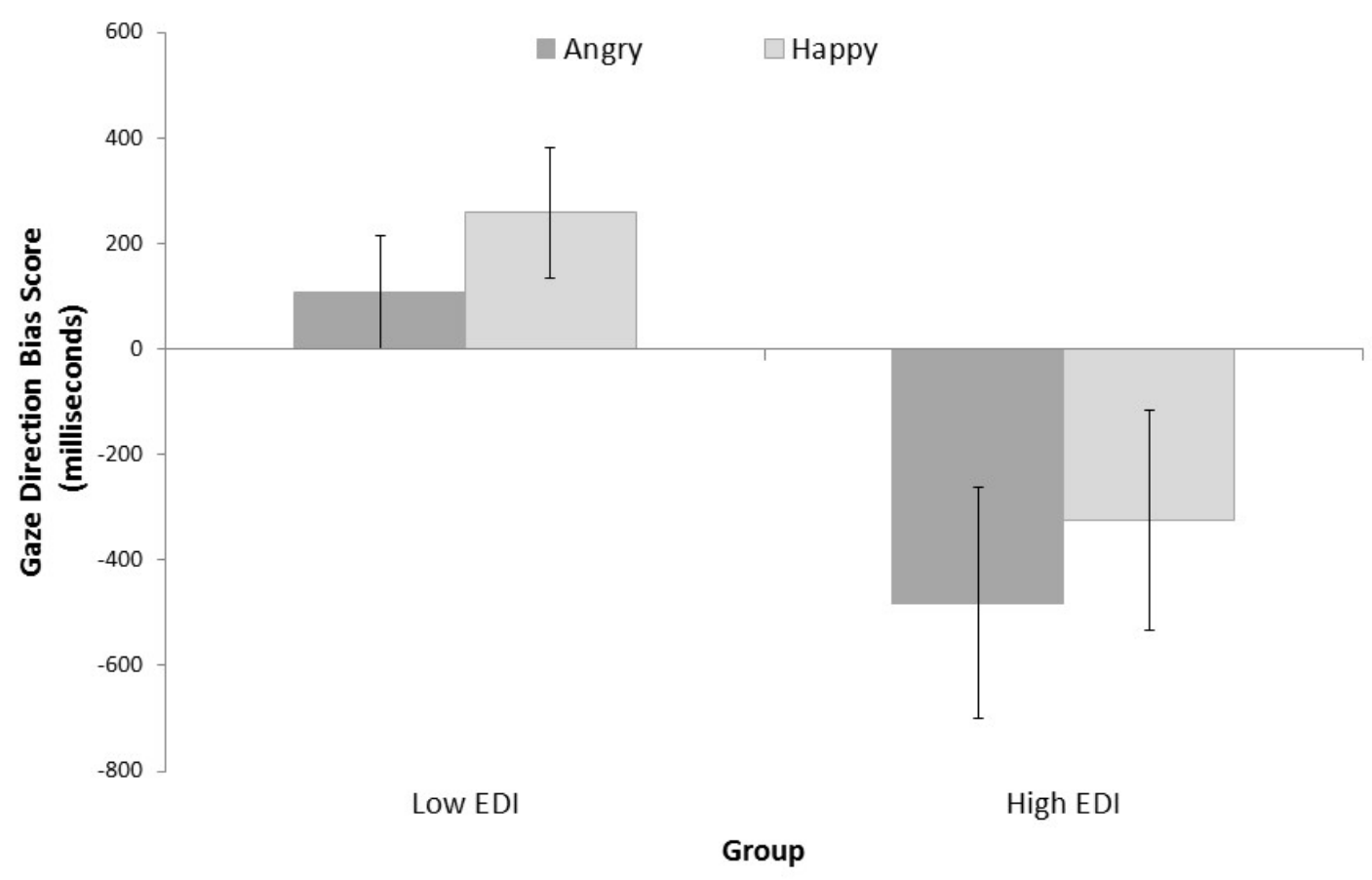

\subsection{Facial emotion recognition (FER)}

Analysis of the participants' emotion recognition accuracy (presented in Table 3) revealed a significant main effect of emotion; $F(6,198)=17.64, p<0.001 ; \eta^{2}{ }^{2}=0.35$. There was also a significant main effect of group, such that participants in the low EDI group correctly recognised a greater percentage of emotional expressions 
(Mean=92.86, SD=12.4) than did participants in the high EDI group (Mean=85.71, $S D=17.8) ; F(1,33)=4.12, p=0.050 ; \eta^{2} p=0.11$. However, this needs to be considered in the light of a significant group $x$ emotion interaction; $F(6,198)=2.53, p=0.022$; $\eta^{2} \mathrm{p}=0.07$. Pairwise comparisons using independent t-tests revealed that the high group was significantly impaired in the recognition of fear and neutral affect; $t(33)=2.09, p=0.045$ and $t(33)=2.92, p=0.01$ respectively. No other differences were significant, all tests $p>.05$.

Table 3: Mean percentage (\%) of emotional expressions correctly recognised as a function of participant group (standard deviations are shown in parentheses)

\begin{tabular}{lccc}
\hline Type of Emotion & Low EDI & High EDI & Total \\
& $(\mathbf{n = 1 7 )}$ & $(\mathbf{n = 1 8})$ & $(\mathbf{n = 3 5 )}$ \\
\hline Disgust & $85.29(29.4)$ & $69.44(34.9)$ & $77.37(32.15)^{\mathrm{dgik}}$ \\
Fear & $76.47(25.7)^{\mathrm{b}}$ & $58.33(25.7)^{\mathrm{b}}$ & $67.4(25.7)^{\mathrm{ehjlm}}$ \\
Happiness & $100(0)$ & $100(0)$ & $100(0)^{\mathrm{def}}$ \\
Surprise & $91.18(19.6)$ & $94.44(16.2)$ & $92.81(17.9)^{\mathrm{kl}}$ \\
Neutral & $100(0)^{\mathrm{c}}$ & $83.33(24.3)^{\mathrm{c}}$ & $91.67(12.15)^{\mathrm{fm}}$ \\
Sadness & $100(0)$ & $97.22(11.8)$ & $98.61(5.9)^{\mathrm{gh}}$ \\
Anger & $97.06(12.1)$ & $97.22(11.8)$ & $97.14(11.95)^{\mathrm{j}}$ \\
\hline Total FER Accuracy & $92.86(12.4)^{\mathrm{a}}$ & $85.71(17.8)^{\mathrm{a}}$ & $89.28(15.11)$ \\
\hline
\end{tabular}

FER=facial emotion recognition

abf $p<0.05$; ck $p<0.01$; deghjlm $p<.001$ 
3.5. The influence of mood, alexithymia and eating-related psychopathology on face processing

Correlational analyses revealed that initial fixation bias scores (direction and duration) were not significantly related to any of the EDI subscales, all tests $p>0.05$. Similarly, gaze duration bias scores were not significantly related to bulimia or body dissatisfaction scores, both tests $p>0.05$. On the other hand, gaze duration scores for both happy and angry expressions were significantly related to scores on the drive for thinness (DFT) subscale of the EDI; $r(52)=-0.35, p=0.011$ and $r(52)=-0.29$, $p=0.038$ respectively, which suggests that higher DFT scores were associated with longer fixation times on neutral expressions compared to emotional. The relationship between DFT and gaze duration for happy expressions remained after controlling for multiple tests (adjusted alpha =0.017), but the relationship between DFT and gaze duration for angry faces was no longer significant. As depression and anxiety have both been shown to influence attentional processing of faces we conducted a hierarchical regression with depression and anxiety entered at the first level and scores on the DFT subscale entered at the second level to determine if DFT score predicted gaze duration on happy faces after controlling for depression and anxiety. The combined contribution of depression and anxiety only accounted for around $5 \%$ of the variance in gaze duration on happy faces; $R^{2}=0.05$, adjusted $R^{2}=0.02$, which was not significant; $F(2,51)=1.4, p>0.05$. Neither depression nor anxiety entered as significant predictors, both tests $p>0.05$. However, the addition of DFT scores produced a significant model that accounted for around $15 \%$ of the variance in gaze duration on happy faces; $R^{2}=0.17$, adjusted $R^{2}=0.12 ; F(3,48)=3.3, p=0.027$, which represented a significant increase in $R^{2}\left(R^{2} \Delta=0.12\right), F(1,48)=6.9, p=0.012$. DFT score entered as the only significant predictor of gaze duration on happy faces, Beta $=-0.47, p=0.012$.

The overall percentage of correctly recognised emotional expressions was negatively related to scores on the DFT and bulimia subscales of the EDI; $r(52)=-$ $0.45, p=0.001$ and $r(52)=-0.30, p=0.033$ respectively, but not the body dissatisfaction subscale, $r(52)=-0.20, p=0.17$. Only the relationship between DFT and emotion recognition accuracy remained once alpha was adjusted for multiple tests $(p=0.017)$. Given that depression, anxiety and alexithymia have been shown to 
impair facial emotion recognition, we conducted a hierarchical multiple regression with depression, anxiety and alexithymia scores entered at the first level and DFT scores entered at the second level in order to determine if scores on the DFT subscale predicted facial emotion recognition after controlling for the other variables. Results revealed that the combined effects of depression, anxiety and alexithymia explained around $10 \%$ of the variance in emotion recognition accuracy, $R^{2}=0.11$, adjusted $R^{2}=0.06$, but this was non-significant; $F(3,48)=1.98, p>0.05$. None of the factors entered as significant predictors, all tests $p>0.05$. However, the addition of DFT scores produced a significant model that accounted for approximately $20 \%$ of the variance in emotion recognition accuracy; $R^{2}=0.22$, adjusted $R^{2}=0.15 ; F(4$, $47)=3.2, p=0.039$, which represented a significant increase in $R^{2}\left(R^{2} \Delta=0.11\right), F(1$, $47)=6.26, p=0.016$. Drive for thinness score entered as the only significant predictor of emotion recognition accuracy, Beta $=-0.44, p=0.016$.

\section{Discussion}

The primary aim of this study was to examine if the bias towards angry faces that has been observed in patients with eating disorders generalised to a non-clinical sample who varied in eating disorder-related symptoms. Furthermore, we examined if the bias related to initial orientation or subsequent disengagement. Finally, we aimed to confirm previous findings of impaired facial emotion recognition in participants with non-clinical levels of eating disorder-related symptoms.

Contrary to our predictions, the high EDI scorers were not more likely to initially orient to angry faces than were low EDI scorers. Furthermore, in comparison to low scorers, the high EDI group did not spend longer looking at the angry faces during their initial fixations. Taken together these findings provide no evidence of an orientation bias towards angry faces in participants who may be considered 'at risk' of developing an eating disorder. This was also confirmed by the continuous analysis, as the location and duration of initial fixations were not related to scores on the EDI subscales. These findings are inconsistent with biases reported in patients with eating disorders (Cardi et al., 2012; Harrison et al., 2010a; Kanakam et al., 2013) and in recovered eating disorder patients (Harrison et al., 2010b) and suggest 
that the bias might not generalise to healthy participants with non-clinical levels of eating disorder-related symptoms.

Contrary to our predictions, the high EDI scorers did not spend longer overall looking at angry faces than did the low EDI scorers, which suggests that the problem in disengaging from threatening faces observed in patients with eating disorders (Cardi et al., 2012) might not generalise to those with non-clinical levels of eating disorderrelated symptoms. Interestingly, low EDI scorers spent longer looking at emotional faces (happy and angry) compared to neutral, whereas the high EDI group spent longer looking at neutral faces compared to emotional. This could be interpreted as avoidance of emotion on the part of the high EDI scorers. This is consistent with the avoidance of positive and negative words observed in participants with non-clinical disordered eating (Seddon and Waller, 2000). It is also consistent with the recent studies reporting that patients with eating disorders avoid looking at emotional faces (Cardi et al. 2015; Davies et al., 2014; Rhind et al., 2014). Continuous analysis confirmed that eating disorder-related symptoms, particularly drive-for-thinness (DFT), was associated with a bias away from the emotional faces. However, the avoidance of angry faces was only a non-significant trend once alpha had been adjusted for multiple tests.

Avoidance of emotional faces (positive and negative) has been observed in participants with social anxiety (Mansell et al., 1999). Therefore, given the evidence of a relationship between drive-for-thinness and social anxiety (Gilbert and Meyer, 2003; Hinrichsen et al., 2004), it is plausible that the bias away from emotional faces exhibited by the high EDI scorers and patients with eating disorders might be a consequence of concomitant social anxiety. However, as we did not obtain a measure of social anxiety, we were unable to confirm this.

In line with our predictions, the high EDI group exhibited relatively impaired facial emotion recognition in comparison to the low group. This is consistent with previous findings in other non-clinical samples (Jones et al., 2009; Ridout et al., 2010; 2012) and patients with clinical eating disorders (Jänsch et al., 2009; Kucharska-Pietura et al., 2004; Pollatos et al., 2008). However, contrary to predictions, this deficit was not more evident for negative emotions; although the high EDI group did recognise significantly fewer fear expressions than did the low EDI group, the largest group 
difference was for neutral expressions. The finding that facial emotion recognition correlated with scores on the EDI subscales is consistent with previous work (e.g. Ridout et al., 2010; 2012). However, our finding that it was drive for thinness and not alexithymia that was the key factor explaining variations in facial emotion recognition is not consistent with previous work (e.g. Brewer et al., 2015). The finding that individuals with non-clinical levels of eating disorder-related symptoms were less accurate than low scorers in recognising emotion from faces is notable, because it might represent a risk factor for the development of eating disorders. Similarly, the tendency to avoid looking at emotional faces could also contribute to the development of eating disorders. For example, lowered sensitivity to, or avoidance of, the facial expressions of significant others could plausibly lead to social misunderstandings, which in turn could result in interpersonal conflicts, undermining important social bonds.

The current study is important because it is the first to examine attention to emotional faces in healthy participants who vary in eating disorder-related symptoms and the first to use eye tracking technology to investigate attentional processing of emotional faces in disordered eating. The use of this technology to continuously monitor the allocation of attention throughout the face processing task is a methodological strength of the current study, as it overcomes some of the limitations of the paradigms (Stroop and dot probe) that have been used in previous studies. The current work is also important because, as noted earlier, research into cognitive deficits in eating disorders has tended to focus on clinical populations, which has made it particularly difficult to separate the causal and maintaining factors implicated in disordered eating (Stice, 2002). The current approach of examining the influence non-clinical levels of eating disorder-related symptoms on face processing has the potential to develop our understanding of possible predisposing factors to eating disorders. For example, this approach might help us to elucidate the nature of symptom development and progression. Furthermore, given that it is known that those in the early stages of eating disorder development are the most likely to respond to treatment (Gordon, 2000), identifying factors involved in the development of disordered eating might help to inform early intervention strategies. 
Although the current findings are novel and important, there were a number of limitations that need to be considered. The participant sample was quite small, particularly for the group analysis. Nevertheless, it is comparable with sample sizes reported in three eye-tracking studies involving participants with clinical (Giel et al., 2011) and non-clinical disordered eating (Gao et al., 2011; Hewig et al., 2008). Furthermore, we replicated the medium effect size for the facial emotion recognition deficit and the large effect size for the attention bias to anger reported in Harrison et al. (2010a), albeit that our attention bias was in the opposite direction to that predicted. A second limitation concerns the number of stimuli included in the face processing tasks. Although the number of trials $(n=24)$ in the facial attention task was broadly similar to Giel et al. (2011), who used 30 picture pairs, the number of trials in the facial emotion recognition task $(n=14)$ was substantially lower than in other similar studies (e.g. Jones et al., 2009), but it was a greater number than that used in the recent study by Cardi et al. $(2015 ; n=4)$. The relatively small number of trials on the facial attention task might have contributed to the large variations in the participants' eye movement data. It is therefore recommended that future studies should include a larger set of stimuli in order to obtain a more reliable estimate of attentional deployment. Nevertheless, this task still proved sensitive to differences between groups of nonclinical individuals who varied within the normal range on a measure of eating disorder-related psychopathology. A final issue concerns the fact that participants were asked to complete the questionnaires, including a measure of depression, before they completed the face processing tasks. It is plausible that asking participants to focus on their mood may have primed negative cognitions. However, it is notable that this procedure is consistent with previous studies looking at attention in participants with eating disorders (e.g. Cardi et al., 2015; Giel et al., 2011). Furthermore, had there been a priming effect it would have been expected that those with higher depression scores would have exhibited an attention bias towards negative stimuli and a recognition bias for negative facial expressions, neither of which were observed.

In conclusion, we confirmed previous findings of less accurate facial emotion recognition in healthy participants with non-clinical levels of eating disorder-related symptoms. We also found evidence of avoidance of emotional faces in these participants. However, our results suggest that the attentional bias towards angry 
faces that has been observed in eating disorders does not appear to generalise to non-clinical eating psychopathology, although caution is required in drawing strong conclusions from the current data given the limitations identified above.

Nevertheless, the current study does demonstrate the potential of using eye tracking to understand changes in attentional processing in eating disorders and of examining non-clinical samples to investigate possible risk factors for eating disorders. It is plausible that the variations in face processing observed in the current sample of healthy participants with relatively high eating disorder-related symptoms might represent a risk factor for the development of eating disorders by disrupting normal social functioning. 


\section{Acknowledgements}

The authors would like to acknowledge Claire Sanderson for her technical support.

\section{Conflict of interest}

The authors have no conflicts of interest to declare.

\section{Role of the Funding Source}

This research was supported by a Loughborough University PhD studentship awarded to the first author. The funder took no role in the development of the research question or the design of the study. 


\section{References}

Bagby, R. M., Parker, J. D., Taylor, G. J., 1994. The twenty-item Toronto Alexithymia Scale-I. Item selection and cross-validation of the factor structure. Journal of Psychosomatic Research, 38(1), 23-32.

Beck, A. T., Steer, R. A., Brown, G., 1996. Beck Depression Inventory II. San Antonio (TX): Harcourt Assessment, Inc.

Brewer, R., Cook, R., Cardi, V., Treasure, J., Bird, G., 2015, Emotion recognition deficits in eating disorders are explained by co-occurring alexithymia. Royal Society Open Science, 2: 140382.

Caglar-Nazalia, H. P., Corfield, F., Cardi, V. Ambwani, S., Leppanen, J., Olabintan, O., Deriziotis, S., Hadjimichalis, A., Scognamiglio, P., Eshkevari, E., Micali, N., Treasure, J., 2014. A systematic review and meta-analysis of systems for social processes in eating disorders. Neuroscience and Biobehavioral Reviews, 42, 55-92

Caivo, M. G., Nummenmaa, L., Hyönä, J., 2007. Emotional and neutral scenes in competition: Orienting, efficiency, and identification. Quarterly Journal of Experimental Psychology, 60, 1585-1593.

Cardi, V., Di Matteo, R., Corfield, F., Treasure, J., 2012. Social reward and rejection sensitivity in eating disorders: An investigation of attentional bias and early experiences. The World Journal of Biological Psychiatry, 14(8), 622-633.

Cardi, V., Corfield, F., Leppanen, J., Rhind, C., Deriziotis, S., Hadjimichalis, A., Hibbs, R., Micali, N, Treasure, J., 2015, Emotional processing, recognition, empathy and evoked facial expression in eating disorders: an experimental study to map deficits in social cognition. PLoS ONE, 10(8): e0133827. doi:10.1371/ journal.pone.0133827

Cooper, R. M., Langton, S. R. H., 2006. Attentional bias to angry faces using the dotprobe task? It depends when you look for it. Behaviour Research and Therapy, 44, 1321-1329.

Davies, H., Schmidt, U., Stahl, D., Tchanturia, K., 2011. Evoked facial emotional expression and emotional experience in people with anorexia nervosa. International Journal of Eating Disorders, 44, 531-539.

Fox, E., Russo, R., Dutton, K., 2002. Attentional bias for threat: Evidence for delayed disengagement from emotional faces. Cognition and Emotion, 16(3), 355-379.

Gao, X., Wang, Q., Jackson, T., Zhao, G., Liang, Y., Chen, H., 2011. Biases in orienting and maintenance of attention among weight dissatisfied women: An eyemovement study. Behaviour, Research and Therapy, 49, 252-259.

Garner, D. M., 1991. Eating Disorder Inventory-2: Professional manual. Odessa, FL: Psychological Assessment Resources. 
Giel, K. E., Friederich, H. C., Teufel, M., Hautzinger, M., Enck, P., Zipfel, S., 2011. Attentional processing of food pictures in individuals with anorexia nervosa- an eyetracking study. Biological Psychiatry, 69, 661-667.

Gilbert, N., Meyer, C., 2003. Social anxiety and social comparison: differential links with restrictive and bulimic attitudes among nonclinical women Eating Behaviors, 4 257-264

Gordon, R. A., (2000). Eating disorders: Anatomy of a social epidemic. Blackwell Publishers Ltd. Pp. 25.

Harrison, A., Sullivan, S., Tchanturia, K., Treasure, J., 2010a. Emotional functioning in eating disorders: attentional bias, emotion recognition and emotion regulation. Psychological Medicine, 40(11):1887-97

Harrison, A., Tchanturia, K., Treasure, J., 2010b. Attentional bias, emotion recognition, and emotion regulation in anorexia: State or trait? Biological Psychiatry, 68(8), 755-761.

Hewig, J., Cooper, S., Trippe, R. H., Hecht, H. D.; Straube, T., Miltner, W. H. R., 2008. Drive for thinness and attention toward specific body parts in a nonclinical sample. Psychosomatic Medicine, 70(6), 729-736.

Hinrichsen, H., Waller, G., van Gerko, K., 2004. Social anxiety and agoraphobia in the eating disorders: associations with eating attitudes and behaviours. Eating Behaviors, 5(4), 285-290.

Jänsch, C., Harmer, C., Cooper, M.J., 2009. Emotional processing in women with anorexia nervosa and in healthy volunteers. Eating Behaviors, 10(3), 184-191.

Jones, L., Harmer, C., Cowen, P., Cooper, M., 2009. Emotional face processing in women with high and low levels of eating disorder related symptoms. Eating Behaviors, 9, 389-397.

Kanakam, N., Krug, I., Raoult, C., Collier, D., Treasure, J., 2013. Social and Emotional Processing as a Behavioural Endophenotype in Eating Disorders: A Pilot Investigation in Twins. European Eating Disorders Review, 21(4), 294-307

Kucharska-Pietura, K., Nikolaou, V., Masiak, M., Treasure, J., 2004. The recognition of emotion in the faces and voice of anorexia nervosa. International Journal of Eating Disorders, 35(1), 42-47.

Laquatra, T. A., Clopton, J. R., 1994. Characteristics of alexithymia and eating disorders in college women. Addictive Behaviours, 19(4), 373-380.

Mansell, W., Clark, D.M., Ehlers, A., Chen, Y.P., 1999. Social anxiety and attention away from emotional faces. Cognition and Emotion, 13(6), 673-690. 
McManus, F., Waller, G., Chadwick, P., 1996. Biases in the processing of different forms of threat in bulimic and comparison women. Journal of Nervous and Mental Disease 184, 547-554.

Mogg, K., Bradley, B. P., Field, M., De Houwer, J., 2003. Eye movements to smoking-related pictures in smokers: Relationship between attentional biases and implicit and explicit measures of stimulus valence. Addiction 98, 825- 836.

Nummenmaa, L., Hyönä, J., Caivo, M. G., 2006. Eye movement assessment of selective attention capture by emotional pictures. Emotion, 6, 257-268.

Pollatos, O., Herbert, B.M., Schandry, R., Gramann, K., 2008. Impaired central processing of emotional faces in anorexia nervosa. Psychosomatic Medicine, 70(6), 701-708.

Quinton, S., Wagner, H. L., 2005. Alexithymia, ambivalence over emotional expression, and eating attitudes. Personality and Individual Differences, 38,11631173.

Rhind, C., Mandy, W., Treasure, J., Tchanturia, K., 2014. An exploratory study of evoked facial affect in adolescent females with anorexia nervosa. Psychiatry Research 15, 711-715.

Ridout, N., Thom, C., Wallis, D., 2010. Emotion recognition and alexithymia in females with non-clinical disordered eating. Eating Behaviors, 11, 1-5.

Ridout, N., Wallis, D. J., Autwal, Y., Sellis, J., 2012. The influence of emotional intensity on facial emotion recognition in sub-clinical disordered eating. Appetite, 59, 181-186.

Russell, T., Schmidt, U., Tchanturia, K., 2009. Aspects of social cognition in anorexia nervosa: affective and cognitive theory of mind. Psychiatry Research, 168, 181-185.

Seddon, K., Waller, G., 2000. Emotional processing and bulimic psychopathology: Age as a factor among nonclinical women. International Journal of Eating Disorders, 28, 364-369.

Spielberger, C. D., Gorsuch, R., Lushene, R., 1970. Manual for the State-trait Anxiety Inventory. Palo Alto (CA): Consulting Psychologist Press.

Stice, E. (2002). Risk and maintenance factors for eating pathology: A meta-analytic review. Psychological Bulletin, 128, 825-848.

Yin, L., Wei, X., Sun, Y., Wang, J., Rosato, M.J., 2006. A 3D facial expression database for facial behavior research. In IEEE 7th International Conference on Automatic Face and Gesture Recognition, Southampton, UK (Vol. 1012, pp. $211-$ 216). 